PAPER

\section{Oscillatory and Chaotic Buoyant-Thermocapillary Convection in the Large-Scale Liquid Bridge}

To cite this article: Jia Wang et al 2017 Chinese Phys. Lett. 34074703

View the article online for updates and enhancements.
Related content

- Instabilities of thermocapillary-buovancy
$\frac{\text { convection in open rectanqular liquid }}{\text { lavers }}$
Huan Jiang, Li Duan and Qi Kang
- Wavenumber Selection by Bénard-
$\frac{\text { Marangoni Convection at High }}{\text { Supercritical Number }}$
Di Wu, Li Duan and Qi Kang
- Space experiment on the instability of
$\frac{\text { Marangoni convection in large liquid bridge }}{\text { - MEIS-4: effect of Prandtl number - }}$
T Yano, K Nishino, H Kawamura et al.




\title{
Oscillatory and Chaotic Buoyant-Thermocapillary Convection in the Large-Scale Liquid Bridge *
}

\author{
Jia Wang(王佳 $)^{1,2}$, Li Duan(段俐 $)^{1,2 * *}$, Qi Kang(康琦 $)^{1,2 * *}$ \\ ${ }^{1}$ Key Laboratory of Microgravity, Institute of Mechanics, Chinese Academy of Sciences, Beijing 100190 \\ ${ }^{2}$ School of Engineering Sciences, University of Chinese Academy of Sciences, Beijing 100049
}

(Received 15 March 2017)

\begin{abstract}
To cooperate with Chinese TG-2 space experiment project, the transition process from steady to regular oscillatory flow, and finally to chaos is experimentally studied in buoyant-thermocapillary convection. The onset of oscillation and further transitional convective behavior are detected by measuring the temperature in large-scale liquid bridge of $2 \mathrm{cSt}$ silicone oil. To identify the various dynamical regimes, the Fourier transform and fractal theory are used to reveal the frequency and amplitude characteristics of the flow motion. The experimental results indicate the co-existence of quasi-periodic and the Feigenbaum bifurcation in chaos.
\end{abstract}

\section{PACS: 47.27.Cn, 47.20.Dr, 47.20.Ky $\quad$ DOI: 10.1088/0256-307X/34/7/074703}

Thermocapillary convection driven by the gradient of surface tension has attracted increasing attention owning to its general application in material science, ${ }^{[1]}$ chemical engineering, and space manufacturing, ${ }^{[2-6]}$ especially the containerless method of high-quality crystal growth. ${ }^{[1,7]}$ Far beyond the critical conditions, it has been pointed out that there are many routes to chaos in thermocapillary convection, depending on the typical parameters, for instance, the Prandtl number, initial disturbance and related geometric parameters. ${ }^{[1]}$ As a paradigmatic example of the nonlinear stability system, the problem of liquid bridge (LB) has been researched numerically ${ }^{[7-9]}$ and experimentally in normal gravity ${ }^{[10]}$ and microgravity conditions. ${ }^{[11]}$

In some ground-based conclusions, the boundary effect in the small-scale one (i.e., diameter is smaller than $6 \mathrm{~mm}$ ) is so obvious that the achievement is incapable of simulating industrial crystal growth effectively. Large-scale LB with large aspect ratio $\left(A_{\mathrm{r}}\right)$ and volume ratio $\left(V_{\mathrm{r}}\right)$ is difficult to establish owing to gravity. In space, large-scale LB with $A_{\mathrm{r}}>1$ and $V_{\mathrm{r}}>1$ can be built due to the decline of gravity. ${ }^{[12]}$ Meanwhile, the thermal convection caused by surface tension which has played a crucial role in space LB experiment can greatly affect the quality of the crystal. Considering the high cost and few opportunities of the space experiments, carrying out the research of large-scale LB with small $A_{\mathrm{r}}$ on the ground in advance is particularly important. ${ }^{[13]}$

On account of its theoretical and application implications, most studies concentrated on the onset of the oscillatory convection, which focus only on the beginning of the route to turbulence and are far from the chaotic state. As a typical dissipative system, when the applied temperature difference continuously increases after the onset of oscillation, the flow may eventually transit to chaos. In this period, the amplitudes of the fluctuation quantities become obviously more nonlinear in their features, and numerous chaos phenomena and related non-linear problems emerge, which is also a very interesting topic in fluid mechanics.

However, due to the flow in LB being very complicated, until 1995, the route of the double-period bifurcation to chaos in LB was confirmed by $\mathrm{Hu}$ et $a l . .^{[10,14]}$ Furthermore, both space and ground experiments are limited to small scale, and the ST-89 have found that the route to turbulent convection depends complicatedly on the temperature difference and geometric parameters. ${ }^{[15]}$ Therefore, the subject on transition to turbulence is still an open subject, and via what route the flow transits to the chaotic state is an interesting question.

In our experiment, the critical conditions have been obtained and discussed by non-dimensional analysis. The route to chaotic convection is presented and focused on the bifurcation characteristics during the transition process. The bifurcation is obtained by the real temporal-frequency analysis and the Fourier transforms.

The LB of silicone oil is floated in the gap between two coaxial rods with the diameter of $20 \mathrm{~mm}$, separated by a distance $H$. To avoid undesired buoyancy convection, the cooling rod is maintained at the ambient temperature by a semiconductor chilling plate. In addition, the geometrical parameters, such as the diameter $d$ of two rods, $A_{\mathrm{r}}=H / d$, and $V_{\mathrm{r}}=V / V_{0}$, are important for the critical point and the transition process. In this experiment, the LB with $A_{\mathrm{r}}=0.18$ and $V_{\mathrm{r}}=0.85$ is studied, where $V_{\mathrm{r}}$ is the ratio of the injection fluid volume $V$ to the volume of the gap $V_{0}$ realized by injection.

Note that the flow field will lose its stability when

* Supported by the China Manned Space Engineering Program (TG-2), the Strategic Priority Research Program on Space Science of Chinese Academy of Sciences: SJ-10 Recoverable Scientific Experiment Satellite under Grant Nos XDA04020405 and XDA04020202-05, and the National Natural Science Foundation of China under Grant No11372328.

** Corresponding author. Email: duanli@imech.ac.cn; kq@imech.ac.cn

(C) 2017 Chinese Physical Society and IOP Publishing Ltd 
the Marangoni number exceeds about 20000, ${ }^{[13,16]}$ and 2cSt silicone oil (Shin-Etsu Chemical Co., Ltd, KF$96 \mathrm{~L}$ ) is selected as the working liquid to provide relatively large Marangoni number. Both rods are designed with a small edge to prevent liquid leakage. To reveal various dynamic states, a temperature acquisition system including a computer, a Keithley 2812 digital multimeter, an Agilent 34970A digital multimeter, and T-type thermocouples are applied to measure temperature signals (shown in Fig. 1). The temperature resolution of the Keithley 2812 is $0.001^{\circ} \mathrm{C}$ and that of Agilent $34970 \mathrm{~A}$ is $0.01^{\circ} \mathrm{C}$. Thermocouples locating on the circumference (diameter is $15 \mathrm{~mm}$ ) of the lower rod are used to measure the inner temperature oscillation, and the sampling rate adopted in this experiment is $10 \mathrm{~Hz}$. The remaining two are used respectively to record the upper heated and lower cooled rods temperature, then feed back to the control sys- tem. The temperature difference controlled by PIDcontrollers (EUROTHERM 3504) with an accuracy better than $\pm 0.05^{\circ} \mathrm{C}$ is applied between the rods made of brass, and by the joint effects of gravity and surface tension, thermal convection will be generated in the liquid zone.

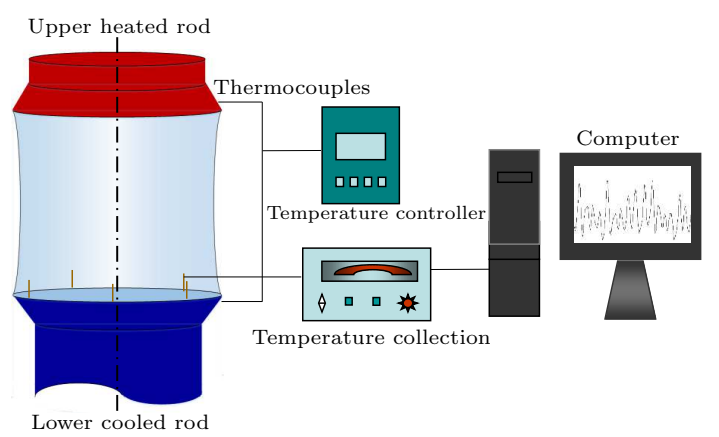

Fig. 1. Schematic diagram of the experimental configuration.

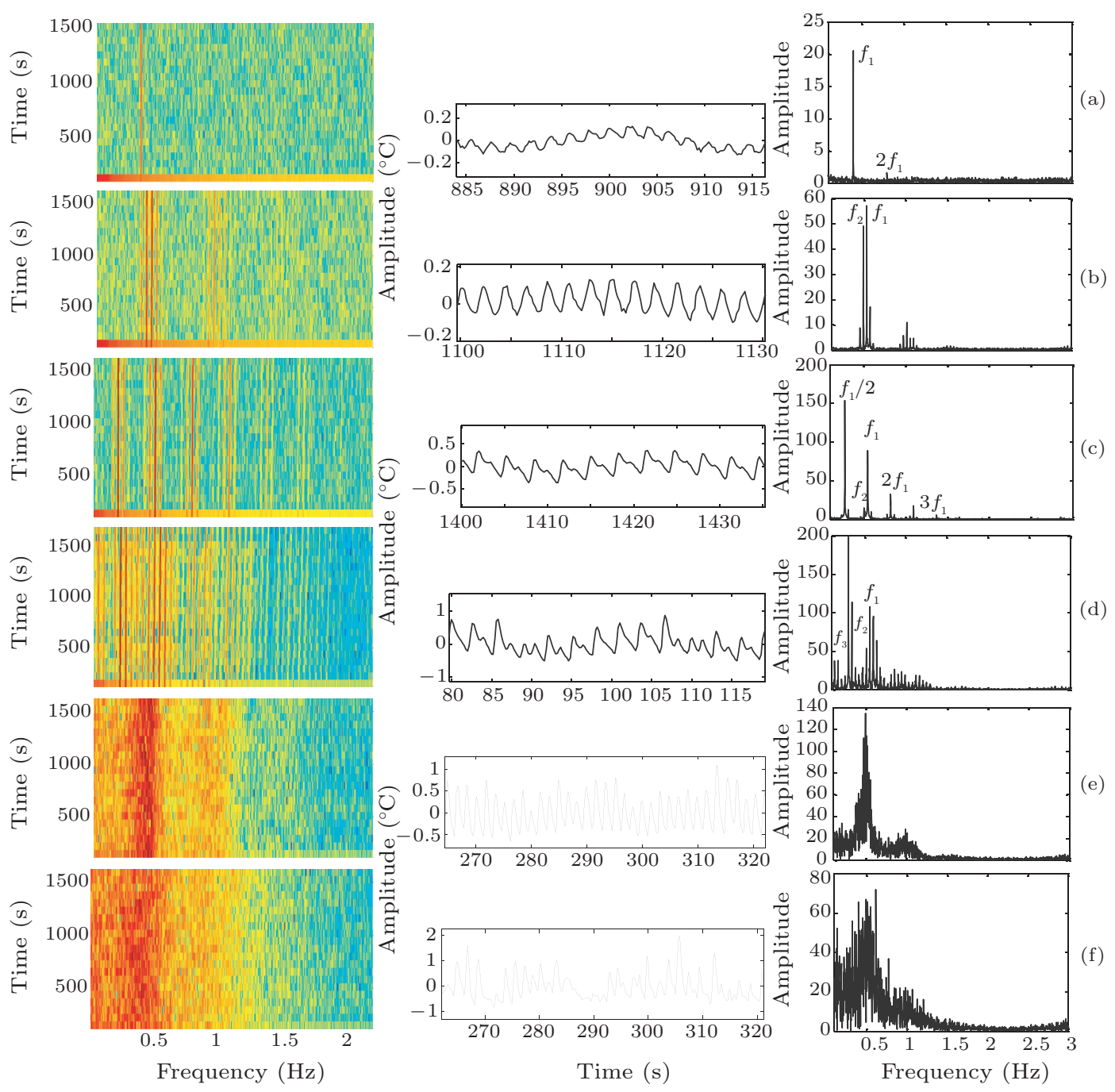

Fig. 2. Temperature records and power spectra showing the sequence of instabilities leading to non-periodic flow. The transition process consists of (1) Rg1: a periodic state with a single peak and its harmonics, $M a=19652$; (2) Rg2: a quasi-periodic state with two incommensurate frequencies $f_{1}$ and $f_{2}$ and their linear combinations, $M a=29479$; (3) Rg3: a state coupled with quasi-periodic and double-period, $M a=37340$; (4) Rg4: a quasiperiodic state with three incommensurate frequencies $f_{1}, f_{2}$ and $f_{3}$ and their linear combinations, $M a=48149 ;(5)$ Rg5: a non-periodic state with relatively sharp peaks just above the onset of noise, $M a=61317$; and (6) Rg6: a strongly non-periodic state with no sharp peaks showing the broadband noise far above its onset, $M a=71143$. 
The applied temperature difference $\Delta T$ between the two ends increases slowly with a heating rate of $0.2^{\circ} \mathrm{C} / \mathrm{min}$. When the oscillation emerges in the temperature field, the temperature difference between the rods holds constant for $20 \mathrm{~min}$ before the next step. To facilitate understanding, $\Delta T$ is non-dimensionalized into the Marangoni number, which can be defined as $M a=\frac{\left|\sigma_{T}\right| \Delta T H}{\rho \bar{v} \alpha}$, representing the strength of the surface tension flow. Figure 2 collects the temperature records, corresponding power spectra and the Fourier spectra under various temperature differences. The increased temperature difference introduces the initial perturbation to the flow field, resulting in the oscillating flow. Above the initial threshold, thermocapillary flow is firstly destabilized to oscillate periodically with finite amplitude and period, which is confirmed by the time history and its spectra with $M a=19652$ in Fig. 2(1). In addition to the fundamental frequency $f_{1}=0.401 \mathrm{~Hz}$, there are also several harmonics at integral multiples of the fundamental one. However, with further increasing $\Delta T$, the nonuniformity of temperature gradient distribution on the free surface is increased, as a result, there is a great temperature gradient in the hot and cold side, and the middle temperature gradient becomes smaller. This change causes the appearance of hot spots and cold spots in LB, which aggravates the instability of the internal flow, leading to the quasi-periodic oscillation in Fig. 2(2). A second fundamental frequency $f_{2}=0.486 \mathrm{~Hz}$ emerges in this regime, and the former one slightly increases to $f_{1}=0.527 \mathrm{~Hz}$. These two frequencies are irreducible, and all the other peaks can be expressed by the linear combination of these two fundamentals.

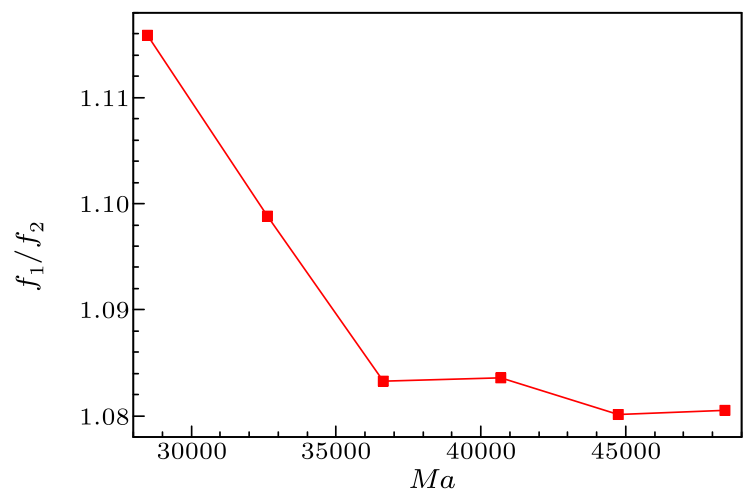

Fig. 3. Frequency ratio $f_{1} / f_{2}$ plotted as a function of $M a$. The ratio has a step indicating phase locking in the range $35000<M a<50000$.

When the Marangoni number is 37340 , the peakto-peak value of single-point temperature is further improved, and the fundamental frequency continues to increase to $0.549 \mathrm{~Hz}$, and the latter one becomes $0.506 \mathrm{~Hz}$. A large number of $k f_{1} / 2(k=1,2,3, \ldots)$ appear in the field, indicating that the quasi-periodic flow and the double periodic flow are coupled with each other in the flow field. The power of $f_{1} / 2$ is much higher than the fundamental ones, and one period of the temperature signal consists of two sub-periods. Phase locking of the two frequencies occurs when $M a$ exceeds 35000. This can be regarded as a step of the ratio $f_{1} / f_{2}$, as shown in Fig. 3 . The ratio decreases smoothly until the step is reached, where the integer ratio $f_{1} / f_{2}=1.080-1.082$. No locking has been observed at lower $M a$, representing that the nonlinear interaction between these oscillations is weak. ${ }^{[17]}$ However, it should be noted that the locking ratio cannot be exactly reproducible as the flow is sensitive to the initial conditions.

A third fundamental frequency appears above this phase locking, as can be seen in Fig. 2(4). In the power spectrum, all the peaks no longer emerge in clusters and can be expressed by the linear combination of three incommensurate frequencies: $f_{1}=0.563 \mathrm{~Hz}$, $f_{2}=0.520 \mathrm{~Hz}$ and $f_{3}=0.133 \mathrm{~Hz}$. As the Marangoni number is increased further to 61317 , the peaks in the power spectrum will become more, and the overlap of these peaks will cause the appearance of some broadened peaks locally. Finally, the broadened peaks will develop to a broadband, which reveals that the flow becomes chaotic.

After the first threshold, the flow experiences the periodic state, quasi-periodic coupled with doubleperiodic state, and finally chaos. During the chaotic route, the flow experiences the quasi-periodic state with three incommensurate frequencies, which is essential for the Ruelle-Takens-Newhouse (RTN) route. However, different from the RTN route in our experiment, the oscillation is coupled with the doubleperiodic one, which is discovered for the first time in the research of transition route to chaos. In addition, rather than becoming chaotic state immediately, the flow will gradually transit to chaos with the development of the signal broadband when the temperature difference is increased further.

The fractal structure and character of the timedependent dynamics of the nonlinear dynamical system can be quantized by fractal number and the Lyapunov exponent, respectively. Both of them will change with the correlative parameters, characterizing quantitatively the evolution of transition process. To distinguish deterministic chaos and random signal, fractal correlation dimension $D_{m}$ in this study is produced by GP algorithm, ${ }^{[18]}$

$$
\begin{aligned}
C_{\mathrm{m}}(r) & =\lim _{N \rightarrow \infty} \frac{1}{N^{2}} \sum_{i, j=1, i \neq j}^{N} \theta\left(r-\left|\nu_{i}-\nu_{j}\right|\right), \\
S\left(\tau_{\mathrm{d}}\right) & =\frac{1}{N} \sum_{i=1}^{N} \log \left(\frac{1}{M} \sum_{k_{i}=1}^{M} \frac{\left|\nu\left(t+\tau_{\mathrm{d}}\right)-\nu\left(t+\tau_{\mathrm{d}}\right)\right|}{\left|\nu(t)-\nu\left(k_{i}\right)\right|}\right)
\end{aligned}
$$

where $\theta$ is the Heaviside function, and by calculating the increase of the averaged divergence rate of the logarithmic distance $S$, we can evaluate the maximum Lyapunov exponent.

The correlation dimension calculation and the maximum Lyapunov exponent presented in Fig. 4 re- 
veal that the thermocapillary flow retain both the fractal structure and the sensitivity to initial conditions of the attractor in the pseudo-phase space. With the increase of the Marangoni number, these two parameters are correspondingly increased. The correlation dimensions increase with different Marangoni numbers, and the mutation of correlation dimension appears when the thermolcapillary convection transits from the stable state to the oscillatory state. Bifurcation of the oscillatory state will lead to mutation of correlation dimension.

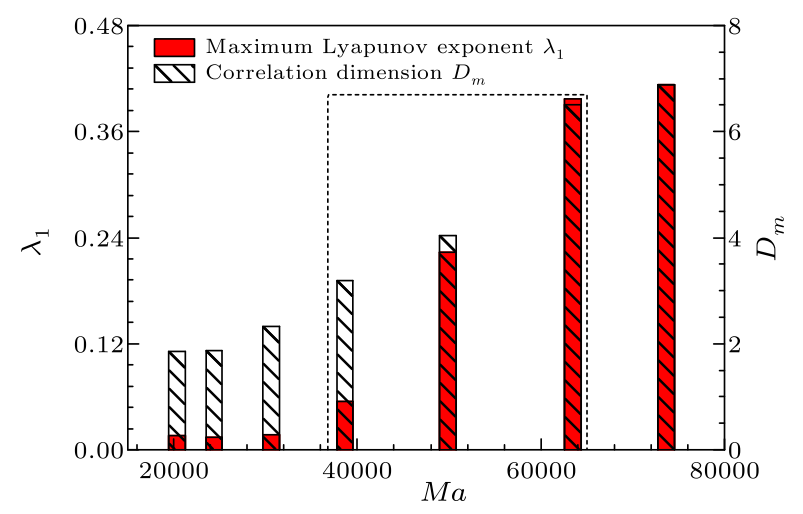

Fig. 4. The relationship between $D_{m}, \lambda_{1}$ and $M a$.

Similar to the experimental results of rectangular liquid pool, ${ }^{[19]}$ there is a mutation in this figure, as shown by the dotted line. Including Rg3-5 in Fig. 2, bifurcations arise frequently in this area. Since the existence of these two parameters leads to important signs to distinguish the chaotic state from the random one, it can be proved that the thermocapillary flow leads to chaos finally.

In conclusion, to cooperate with the TG-2 space experiment, ground experimental study which mainly concentrates upon the convection from steady to the chaotic state in large scale LB has been carried out. A novel transition route that coupled with quasi-periodic and double-periodic states is found. By analyzing the Lyapunov exponent and correlation dimension, it shows that the oscillatory convection becomes chaos eventually, and the bifurcation causes the mutation of these related parameters. These ground experimental results can provide scientific reference and comparison for the space experiment.

\section{References}

[1] Hu W R and Tang Z M 2003 Floating Zone Convection in Crystal Growth Modeling (Beijing: Science Press)

[2] Kang Q, Duan L, Zhang L, Yin Y L, Yang J S and Hu W R 2016 Microgravity Sci. Technol. 28123

[3] Liu Q S, Zhou B H, Nguywn T H and Hu W R 2004 Chin. Phys. Lett. 21686

[4] Zhang S T, Duan L and Kang Q 2016 Exp. Fluids 57113

[5] Liu R, Liu Q S and Hu W R 2005 Chin. Phys. Lett. 22402

[6] Duan L, Kang Q and Hu W R 2008 Chin. Phys. Lett. 25 1347

[7] Li K, Xun B, Imaishi N, Yoda S and Hu W R 2008 Int. J. Heat Fluid Flow 291190

[8] Tang Z M and Hu W R 2003 Chin. Phys. Lett. 20526

[9] Chen Q S and Hu W R 1998 Int. J. Heat Mass Transfer 41 825

[10] A A Y, Cao Z H and Hu W R 2007 Chin. Phys. Lett. 24 475

[11] Kawamura H, Nishino K and Mastumoto S 2012 J. Heat Transfer 134031005

[12] Taishi Y, Koichi N and Hiroshi K 2012 Exp. Fluids 539

[13] Wang J, Wu D, Duan L and Kang Q 2017 Int. J. Heat Mass Transfer 1082107

[14] Tang Z M and Hu W R 1995 Int. J. Heat Mass Transfer 383295

[15] Koichi N, Taishi Y, Hiroshi K, Kawamura H, Matsumoto S, Ueno I and Ermakov M K 2015 J. Cryst. Growth 42057

[16] Schwabe D and Frank S 1992 Exp. Fluids 23234

[17] Gollub J P and Benson S V 1980 J. Fluid Mech. 100449

[18] Ueno I, Tanaka S and Kawamura H 2003 Phys. Fluids 15 408

[19] Zhu P, Duan L and Kang Q 2013 Int. J. Heat Mass Transfer $\mathbf{5 7} 457$ 